\title{
Una de conejos. Análisis estadístico para la creación de juegos de azar para niños
}

\author{
María de las Mercedes Rodríguez Hernández ${ }^{1}$ y Pedro Tadeu ${ }^{2}$ \\ ${ }^{1}$ Universidad de Salamanca (España); ${ }^{2}$ Instituto Politécnico da Guarda (Portugal)
}

La literatura estudia desde las primeras edades hasta las edades adultas las intuiciones que tiene una persona sobre el lanzamiento de monedas. Estos trabajos concluyen que cuando a los alumnos se les manda escribir una secuencia o analizar una secuencia dada que pudiera ser aleatoria, consideran que debe haber un número casi igual de caras y cruces. ¿Quién de nosotros no escribiría una secuencia de esta forma? Cuando tienen que manifestarse respecto a otras características como son las rachas, piensan que en longitudes de rachas cortas y por lo tanto en un número de rachas amplio. ¿Sabemos acaso cuáles son el valor mínimo y máximo o al menos más habitual del número de rachas y de las longitudes de rachas? Este trabajo profundiza en estas preguntas desde diferentes perspectivas.

Palabras clave: Lanzamiento de monedas, azar, tamaño de secuencias, equiprobabilidad, Ley de los Grandes Números.

One about rabbits. Statistical analysis to creation of games of chance for children. The literature studies from the first ages to adult the intuitions that a person has about the coin toss. These works conclude that when students are instructed to write a sequence or analyze a given sequence that could be random, they consider that there should be an almost equal number of faces and crosses. Who among us would not write a sequence this way? When they have to manifest themselves with respect to other characteristics such as streaks, they think that in short streak lengths and therefore in a wide number of streaks. Do we know what are the minimum and maximum or at least more usual value of the number of streaks and the lengths of streaks? This work deepens these questions from different perspectives.

Key words: Coin toss, random, sequence size, equiprobability, the Law of Big Numbers.

Correspondencia: $\mathrm{M}^{\mathrm{a}}$ Mercedes Rodríguez Hernández. C/ San Pedro del Barco $\mathrm{N}^{\mathrm{o}} 72^{\circ}$ 3. C.P.: 05003 Ávila, Ávila (España). E-mail: mercedes.r.h@ hotmail.com 
En muchas de las situaciones de la vida real se deben tomar determinadas decisiones, para lo que es importante evaluar los distintos resultados que pueden ocurrir. Las posibilidades de elección de un acontecimiento pueden variar enormemente, además pueden suceder con mayor o menor grado.

El vocabulario de la probabilidad utiliza expresiones como "imposible", "posible”, "casi seguro”, etc., pero que significa ¿muy probable? o ¿muy posible? esto puede diferir en la opinión de un individuo a otro, es por esto, que se utilizan números para cuantificar una determinada ocurrencia. La probabilidad varía entre 0 y 1 . La no ocurrencia de una situación tiene probabilidad nula. La probabilidad 1, equivale a la ocurrencia segura. ninguna niña.

El Sr y Sra Buenaesperanza esperan un nuevo bebé. Tienen tres niños y

Sra Buenaesperanza: ¡Cuánto espero que nuestro próximo bebé sea niña!

Señor Buenaesperanza: Querida, después de tres niños, no sé qué pensar.

- Por una parte, parece que tenemos más posibilidades de que sea otro niño. Se nos da bien tener niños.

- Por otra, como ya hemos tenido tres niños seguidos, diría que es casi forzoso que ahora sea niña.

Si usted piensa afirmativamente cualquiera de estas dos afirmaciones, ha caído en lo que se llama la falacia del jugador. La probabilidad no depende del acontecimiento ocurrido. Las posibilidades de que los Buenaesperanza tengan otro niño es la misma que la de que su primer hijo lo fuera. Para mejorar esta aclaración, supongamos que los Buenaesperanza lanzan una moneda equilibrada y sacan tres caras seguidas. La probabilidad de que en un nuevo lanzamiento la moneda salga otra vez cara es idéntica a la de los lanzamientos anteriores. Hay un 50\% de posibilidades de que salga cara o cruz. La moneda no tiene memoria de lo que sucedió.

El estudio de la probabilidad, a través de un análisis estadístico tanto numérico como gráfico, permite a los alumnos, los que serán futuros ciudadanos adultos, obtener unos conocimientos para poder tomar decisiones, adquirir intuiciones correctas y establecer unos cimientos sólidos de los conocimientos básicos en probabilidad.

Actualmente la enseñanza de la probabilidad en las aulas por los maestros se hace desde el aprendizaje de fórmulas. Se puede aplicar fácilmente y no requieren técnicas matemáticas complicadas. Esto hace que no se comprenda claramente y se olvide con facilidad. En los currículos cada vez se da más importancia a sus aplicaciones. Proporcionan una buena oportunidad para demostrar a un estudiante su utilización para resolver problemas reales o analizar estudios donde se puedan dar diversas situaciones en una mayor o menor medida.

Los maestros no deben ser los simples transmisores del conocimiento, sino que serán los gestores de éste, permitiendo a los alumnos asentar unas bases de 
aprendizaje correctas para poder progresar en sus conocimientos. Utilizar el juego como instrumento es una herramienta ideal para que los niños adquieran las nociones de probabilidad y asienten unas bases sólidas.

Investigaciones psicológicas sobre el razonamiento humano en situaciones de incertidumbre muestran que las intuiciones en el campo de la probabilidad y la estadística nos engañan con frecuencia (Kahneman, Slovic, y Tuerskv, 1982). A diario se toman decisiones basadas en la evaluación de probabilidades para tomar decisiones que en muchas ocasiones no son correctas o están sesgadas. Fischbein (1987), considera las intuiciones como parte de la conducta inteligente, por lo que recomienda que antes de introducir un concepto nuevo de una cierta materia, se realice una investigación sobre los conocimientos intuitivos de los alumnos. Incluso los alumnos llegan a la universidad con conocimientos e intuiciones sobre probabilidades escasas e incorrectas, como manifiesta Carrera (2002).

Numerosos autores hacen estudios en niños donde les proponen un número de lanzamientos de monedas. No explican el porqué de la elección de esos lanzamientos. Green (1983) realiza tareas con alumnos donde les piden que obtengan secuencias de 50 lanzamientos. También les piden elegir entre dos patrones con unas secuencias de 150 lanzamientos cada uno de ellos, para que eligieran cuál creían ellos que eran aleatorio. Arteaga, Ortiz, y Batanero (2008) utiliza 20 lanzamientos, sin justificar la elección de esta cifra numérica. ¿Es suficiente estos números de lanzamientos? ¿Podrá verse el comportamiento de las reglas de azar? ¿Se obtiene una información suficiente para las rachas o las longitudes de las rachas con estos tamaños? Ninguno de estos autores justifica el porqué de la elección de esos lanzamientos.

Existen investigaciones sobre razonamientos probabilísticos que sugieren que la realización de las predicciones y la comparación de los datos obtenidos experimentalmente no son suficientes (Konold, 1995). Para que los estudiantes cambien sus creencias aparte de ver los diferentes resultados es preciso estudiar otras propiedades como la variabilidad o las rachas.

Green (1991) investiga con niños de 11 y 16 años donde prueban que los niños eran muy precisos para reflejar la equiprobabilidad, pero los niños reflejan en conjunto rachas demasiado corta respecto a un conjunto de experimentos del lanzamiento de un proceso aleatorio. ¿Quién de nosotros no escribiría una secuencia con características cercanas a la esperanza matemática?, esto es, cuasi-equiprobable o con un número de rachas ni muy grande ni muy pequeño y además con longitudes de rachas relativamente cortas. Son aspectos diferentes el escribir sólo una secuencia por individuo que al mismo individuo se le pida escribir un conjunto de posibles secuencias que puedan darse al lanzar una moneda, quizás escribiría secuencias con diferente variaciones. 
El estudio llevado a cabo por Green (1990) manifiesta que la percepción de la aleatoriedad no mejora con la edad. Estas conclusiones también pueden verse en Batanero y Serrano (1999). La percepción subjetiva de la aleatoriedad en adultos ha sido investigada en los últimos años por Bar-Hillel y Wageraar (1991) donde se les pide que escriban una secuencia de resultados que se esperaría al lanzar una moneda y también reconocer si la secuencia dada es o no aleatoria. Los adultos rechazan las secuencias de rachas largas. En general los sujetos tienen demasiadas alternancias y rachas cortas.

Lo que nosotros aportamos con este estudio es un análisis de lo que realmente ocurriría para que el docente elija un determinado tamaño adecuado del número de lanzamientos y pueda observar el comportamiento real de las monedas. Analizamos la secuencia de una moneda con un máximo de 40 lanzamientos. Se estudia la equiprobabilidad, cómo y cuánto varían las características de las cadenas: proporción de rachas, número de rachas y longitud de rachas.

El propósito exactamente de este estudio es analizar las diferentes regularidades del lanzamiento de 40 monedas, el número de caras y cruces, el número de rachas, la longitud de las rachas, la proporción de las rachas en una secuencia.

Es importante saber los efectos que tiene el tamaño de la muestra, por ello los objetivos de nuestro estudio son dos:

- Llevar a cabo una simulación computacional para estudiar las secuencias de este tamaño para un número grande de lanzamientos. Conocer las características en las secuencias de 40 lanzamientos.

- Plantea una práctica para los niños, donde a través del juego adquieran las características del lanzamiento de monedas.

Con la que los maestros alcanzarían los siguientes objetivos en el aula:

- Acercarse al azar.

- Simular procesos aleatorios con monedas.

- Realizar observaciones sobre las características de las secuencias obtenidas.

- Practicar la toma de decisiones.

- Hacer predicciones lo más cercanas a la realidad.

\section{METODOLOGÍA}

Se lleva a cabo un estudio de simulación computacional sobre el lanzamiento de monedas. A continuación se explica en que consiste el algoritmo.

Se genera una secuencia de tamaño cuarenta " 0 's" y " 1 's" procedentes de una distribución uniforme. Se calcula la frecuencia relativa de los 0 y 1 . Se calcula la longitud de las rachas y el número de rachas. Se realizan $N$ simulaciones. 
Esta información permitirá tener un estudio estadístico del comportamiento de las secuencias de tamaño 40.

A continuación, se propone una práctica, para niños de 11-12 años, basada en una lectura. Deberán observar la equiprobabilidad, el número de rachas y la longitud de rachas a través del lanzamiento de una moneda. El juego se reducirá a un sencillo recuento para completar una tabla sin mencionar que estamos trabajando con frecuencias o con probabilidades. Antes de comenzar el juego deben hacer determinadas apuestas a los que ellos creen que saldrá, realizan la primera jugada y observan el resultado; apuestan nuevamente y vuelven a jugar, así durante 10 partidas. El motivo es que ellos mismos vean cómo va cambiando su pensamiento a medida que observan los lanzamientos. Se intenta que observen y vayan variando sus decisiones según aumenta el número de partidas, al ir observando ciertas regularidades.

\section{Aplicación práctica}

Se presenta un pequeño texto que se les entrega a alumnos de sexto de Educación Primaria (E.P.).

Texto. Al calcular probabilidades es fácil despistarse. Veamos aquí a una pareja de conejos que se fueros de picos pardos y al cabo de un mes...

Sr Conejo: Oye, salada remilgada ¿Cuántos gazapos hemos tenido en esta camada?

Sra Coneja: ¡Mi presuntuoso Conejo! Basta saber contar. ¡Pues 4!

Sr Conejo: ¿Cuántos son macho?

Sra Conejo: Es difícil saberlo en recién nacidos. No te sientas enojado. No es muy probable que los cuatro hayan sido machos.

Sr conejo: Mi Bella coneja, tampoco lo es que las 4 sean conejas. A lo mejor sólo hay una hembra.

Sra Conejo: Mi querido galán, o tal vez haya solamente un macho.

Sr Conejo: Calculando... que el gazapo sea hembra o macho es cosa de cara o cruz. Así que como lo esperado es que haya 2 machos y 2 hembras, este caso con el número empate debe ser el de mayor posibilidad.

Sr Coneja: Te equivocas Sr Conejo. Si escribes los 16 casos posibles de obtener cara y cruz en el lanzamiento de 4 monedas y piensas un poco, verás que es mayor el número de casos de tener diferente número de hembras y machos, es más fácil tener 3 machos y 1 hembra o 3 hembras y 1 macho (8 casos de los 16) que el de tener 2 hembras y 2 machos (6 casos de los 16).

Ejercicio. Describa los distintos casos de obtener conejitas o conejitos en una camada de 4. 
Lectura. A continuación, se presenta una lectura para posteriormente trabajar sobre esta titulada "los conejos":

La reproducción de los conejos tiene lugar con mucha facilidad y en cualquier época del año, el período gestacional es sumamente corto y las camadas suelen ser bastante numerosas. Por regla general todas las especies de conejos son capaces de reproducirse antes de cumplir un año de vida, la mayoría a los 9 meses. Algunas pueden comenzar incluso antes, a los 4 o 5 meses. En cualquiera de las especies las hembras demoran menos tiempo para estar preparadas físicamente para reproducirse que los machos. Esto ha hecho que en algunos países haya plagas. Pasados los 4 o 5 años de vida el conejo se vuelve demasiado viejo por lo no es recomendable que se continúe apareando.

El período de gestación de los conejos es bastante efímero, siendo considerado uno de los más cortos de todo el reino animal. Una conejita está embarazada entre 29 y 35 días, con un término medio de 31 días. Los síntomas del embarazo de las conejas también pueden ayudar a confirmar su estado, aunque hay algunas que no muestran ningún signo hasta minutos antes de nacer las crías. Normalmente se vuelven bastante arisca con el macho y empiezan a beber más agua de lo normal. Su abdomen empieza a crecer a la par que los embriones y en consecuencia preferirá dormir de lado para tener mayor comodidad. Las mamas también se empiezan a hinchar cuando la producción de leche se inicia. Y cuando faltan unos pocos días para el alumbramiento comenzará a arrancarse el pelo del vientre y lo utilizará para preparar el nido.

La preparación del nido usualmente corre por parte de la coneja, ella buscará todo tipo de cosas que le ayuden con este fin como paja, papel, trapos, toallas o calcetines. Además con el pelo que arranca de su cuerpo acondicionará el nido para que sea más cálido, algo fundamental ya que los conejitos neonatos no son capaces de regular su temperatura hasta los 7 días de nacido.

El nacimiento de todas las crías suele ser bastante rápido, aunque hay partos que se extienden y pueden durar hasta dos días. Los conejitos neonatos son llamados gazapos, nacen sin pelos y completamente ciegos.

Los conejos pueden tener entre 4 y 12 crías por cada camada. Los conejos de campo suelen tener camadas de 3, 4 y 5 gazapos. Los que se encuentran en granjas de cría este número es mucho mayor siendo alrededor de 9 una cifra bastante habitual. Como pueden tener varias camadas en el año el número de crías puede ascender hasta 80 en este tiempo, una cantidad bastante impresionante para su diminuto tamaño.

Una vez que la coneja ha dado a luz su cría, deberás tener mucho cuidado de que el macho no se le acerque a ella o a los conejitos neonatos. Esto es debido a que es posible que la coneja pueda volver a ser mamá inmediatamente después del parto, pero si esto sucede le será imposible amamantar a los gazapos durante todo el período de lactancia. Lo ideal será esperar al menos 40 días antes de quedarse embarazada de 
nuevo. La recién estrenada mamá comenzará a cuidar de su camada y a alimentarla con su leche unos pocos minutos después del nacimiento. Lo usual es que los visite dos veces al día para amamantarlos, generalmente temprano en la mañana y tarde en la noche. La leche materna va a ser el alimento exclusivo de los gazapos hasta el día 18 aproximadamente, luego podrás comenzar a alimentarlos con pienso especial y heno. Para comenzar a darles verdura si deberás esperar a que cumplan al menos los 3 meses de edad.

Así, como las gestaciones son de unos 31 días, la lactancia de unos 56 días, esto hacen un total de 87 días, supongamos entonces que se han dado 4 camadas de 10 gazapos cada una, esto es que tenemos un total de 40 conejitos al año.

A continuación proponen una actividad, en dos partes, para llevar a cabo en el aula.

Se necesita el siguiente material:

- Una hoja cuadriculada. Selecciona 10 filas de 40 cuadritos cada una.

- Una moneda para realizar lanzamientos. Cara simulará el nacimiento de un conejito hembra y cruz el nacimiento de un conejito macho.

Primera parte de la actividad: Cada alumno hará una apuesta del número de veces que cree que saldrá cara en una secuencia de 40 tiradas. Marcando con una x en la primera columna de la tabla 1 . Seguidamente procederá al lanzamiento de una moneda hasta tener una secuencia de 40 lanzamientos que deben rellenar en los cuadritos. Observa el número de caras (conejas), y señala con una x la segunda columna de la tabla 1. Se llevarán a cabo 10 partidas.

Tabla 1. Registro del lanzamiento de 40 monedas

\begin{tabular}{lllll}
\hline \multirow{2}{*}{ Número de hembras por camada } & & & \\
& & & & \\
\hline 10 & & & \\
\hline 11 & & & \\
\hline
\end{tabular}

De esta forma los alumnos irán variando su intuición a medida que avance el juego y el maestro podrá ver como evoluciona su pensamiento.

Se llevaran a cabo otras dos tablas similares a esta una con las rachas de las secuencias y otra con la longitud de rachas.

Segunda parte de la actividad: En esta segunda parte, se pondrán los datos en común en la pizarra. Se hará un recuento del número de conejitas en una camada y se recogerán en la tabla 2 . 
Tabla 2. Recogida de datos de todos los alumnos del aula

\begin{tabular}{ll}
\hline Número de hembras & \\
\hline En una camada de 40 crías & Número de camadas \\
\hline \\
\hline \\
\hline Número de hembras & \\
\hline En una camada de 40 crías & Número de camadas \\
\hline
\end{tabular}

A continuación se plantean las siguientes actividades.

Representa un diagrama de barras de los datos obtenidos. Contesta las siguientes preguntas:

- ¿Cuál es el número de mínimo de hembras obtenido en las camadas? (ver la tabla)

- ¿Cuál es el número de máximo de hembras obtenido en las camadas? (ver la tabla)

- ¿Cuál es el número máximo de hembras seguido de una camada (longitud máxima de las rachas) obtenidas en tu grupo?

- ¿Cuál es el número de las rachas obtenidas en las 10 camadas (de los 40 gazapos) obtenidas en tu grupo? Relaciona este número de forma aproximada con el número de gazapos de la camada.

\section{RESULTADOS}

Se procedes a describir los resultados de 10000 simulaciones de secuencias de tamaño 40, obtenidos a través de del programa computacional.

La proporción de caras que se da con mayor frecuencia es de $\mathrm{Mo}=1 / 2$. El 50\% de los valores, entre el primer cuartil (Q1) y el tercero (Q3), oscila entre 0.45 y 0.55 . Solamente en un $5 \%$ de las ocasiones la proporción de caras es mayor de 0.62 . La tabla 3 de proporciones que se muestra debajo. El histograma de las proporciones de las caras en las secuencias de 40 lanzamientos sigue una distribución normal cuya media es de 0.5 y la desviación típica de $\mathrm{s}=0.07$, como puede verse en el gráfico 1 arriba a la izquierda.

Tabla 3. Proporción de caras en secuencias de tamaño 40

\begin{tabular}{lcc}
\hline Mo & Q1 & Q2 \\
\hline $1 / 2$ & $9 / 20$ & $1 / 2$ \\
\hline Mo & Q1 & Q2 \\
\hline
\end{tabular}


El número de rachas que se da con mayor frecuencia es $20, \mathrm{Mo}=20$, siendo el mínimo de $8, \operatorname{Min}=8$ y el máximo de 31, Max=31, el $50 \%$ de las secuencias oscila entre Q1=17 y Q2=22. Sólo un 10\% de las secuencias hay más de 23 rachas (Tabla 4). La distribución es normal como se aprecia en gráfico 1, arriba a la derecha, con media 19.48 y desviación típica 3.13.

Tabla 4. Características de las rachas en secuencias de tamaño 40 lanzamientos

\begin{tabular}{lccccc}
\hline Número de rachas & Min & Mo & Max & Q1 \\
\hline Proporción de las rachas & 8 & 20 & 31 & 17 \\
\hline Longitud de las rachas & $1 / 5$ & $1 / 2$ & $31 / 40$ & $17 / 40$ \\
\hline Número de rachas & 1 & 1 & 16 & 1 \\
\hline Proporción de las rachas & $1 / 5$ & $1 / 2$ & $31 / 40$ & $17 / 40$ \\
\hline
\end{tabular}

El histograma de la proporción de las rachas se ve en el gráfico 1, abajo a la izquierda, cuya media es 0.48 y desviación típica 0.078 y los datos relativos a las proporciones de las rachas pueden verse en la tabla 4.

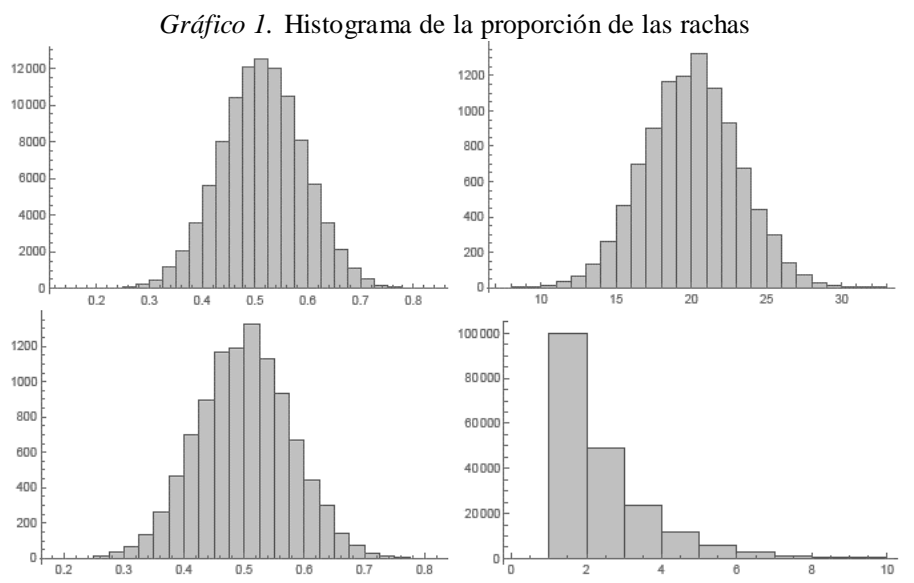

Nota: (Eje Y) Número de simulaciones. (Eje X) Frecuencia relativa del número de caras (arriba-izquierda), número de rachas (arriba-derecha), proporción rachas (abajo-izquierda) y longitud rachas (abajo a la derecha).

Los valores obtenidos de las longitudes de las rachas son normalmente de 1 , sólo un $25 \%$ de los valores son mayores de dos y sólo hay un $1 \%$ de los valores que son mayores de 7, como se muestra en la tabla 4 en el cuartil 3 y el percentil 99. El histograma presenta una asimetría a la derecha, como puede verse en el gráfico 1, abajo a la derecha. 
Batanero y Serrano (1999) piden a los alumnos al mostrarles cuatro secuencias de lanzamientos de 40 monedas que dieran su opinión sobre si creían que las secuencias eran aleatorias o por el contrario veían algo análogo en ellas que les hiciera pensar que no representaban un lanzamiento real. No realizan un estudio de los 40 lanzamientos ¿Cómo saber las características de las secuencias? ¿Cuál es el número que hace que este dentro de los valores de aleatoriedad? A continuación analizamos las secuencias en base al estudio realizado de simulación y además llevamos a cabo el contraste de hipótesis de aleatoriedad.

La primera secuencia que presentan es:

$+++\mathrm{c}+\mathrm{cc}+++\mathrm{c}+\mathrm{ccccc}+\mathrm{c}+\mathrm{c}++\mathrm{c}+\mathrm{cc}++++\mathrm{ccc}+\mathrm{cc}+\mathrm{cc}$

El número de rachas de la secuencia es de 22, igual al Q3 y la proporción de las rachas es de 0.55 por debajo del $\mathrm{Q} 3=0.55$. El valor máximo de las longitudes de rachas es 5 que corresponde al P95=5. La proporción del número de caras en la secuencia es de 0.525 menor que Q3=0.55. Una vez realizado este análisis todo parece indicar que se encuentra dentro de los valores posibles. A continuación, se lleva a cabo un contraste de hipótesis donde la hipótesis nula, afirma que la secuencia es aleatoria, frente a la hipótesis alternativa donde se asegura que la secuencia no es aleatoria. Se concluye que no hay evidencias para rechazar la hipótesis nula. La secuencia no presenta indicios de que tenga alguna irregularidad.

La segunda secuencia es:

$\mathrm{c}+\mathrm{c}++\mathrm{cc}+\mathrm{c}+\mathrm{cc}++\mathrm{c}++\mathrm{cc}++\mathrm{c}+\mathrm{cc}++\mathrm{c}+\mathrm{c}+\mathrm{c}+\mathrm{c}+\mathrm{c}++\mathrm{c}+$

El número de rachas de la cadena es $30<27$. La proporción de las rachas es de $0.75>\mathrm{P}\{99\}=0.67$. La longitud de las rachas máximas es de $2<\mathrm{Q} \_3=2$. La proporción del número de caras en la secuencia mostrada es de $0.475<$ Q_2 $=0.5$. Sólo hay un $1 \%$ de las secuencias que son lanzadas por una moneda que tienen un número de rachas mayor de 27 y por lo tanto una proporción de rachas mayor a 0.67. Lo normal es que el número de rachas oscile en torno a la mitad del tamaño de la secuencia esto es en torno a 20. ¿Cuándo es son demasiadas rachas o demasiado pocas? El P1 indica que sólo hay un $1 \%$ de secuencias que tienen un número menor de rachas y el percentil P99 indica que sólo hay un $1 \%$ de secuencias que tienen un número de rachas mayor. Se efectúa un contraste de hipótesis para estudiar la aleatoriedad de la secuencia. El número de caras y cruces es, nc=19 y n+=21\$ por lo tanto la media es de $20.95 \$$ y la desviación típica de 3.11. En la región crítica por lo tanto se aprecia evidencias de no aleatoriedad, tal como se preveía. 
La tercera secuencia que emplean es:

$\mathrm{c}+++\mathrm{c}++\mathrm{ccc}+\mathrm{c}+++++\mathrm{c}+\mathrm{c}+\mathrm{cc}+\mathrm{c}++\mathrm{cccc}+++\mathrm{c}++\mathrm{ccc}$

El número de rachas es de $21<\mathrm{Q} 3=22$, la proporción de rachas es de $0.525<$ $\mathrm{Q} 3=0.55$. La longitud de las rachas máximas es de $5<\mathrm{P} 95=5$. La proporción de caras en la secuencia es de $0.475<\mathrm{Q} 2=0.5$. Así, que no presenta ningún valor que se dé con poca frecuencia. Realizamos el contraste de hipótesis, donde $n c=19$ y $n+=21$ por lo tanto la media es de 20.95 y desviación típica de 3.11 que se encuentra dentro del intervalo de la región de aceptación para un nivel de significación del 5\%, por tanto no se encuentran evidencias para rechazar la aleatoriedad.

La cuarta secuencia es:

$\mathrm{c}+++\mathrm{c}++\mathrm{c}+\mathrm{c}+++\mathrm{c}++++\mathrm{cc}+++\mathrm{c}++\mathrm{c}++\mathrm{c}++++\mathrm{c}+++\mathrm{c}+$

El número de rachas es $22<\mathrm{Q} 3=22$, la proporción de las rachas es $0.55<$ $\mathrm{Q} 3=0.55$. L longitud máxima de las rachas es de $4<\mathrm{P} 90=4$ y la frecuencia relativa de caras es $0.3<\mathrm{P} 1=0.325$, el $1 \%$ de las secuencias en 40 lanzamientos se encuentran en una proporción menor que $\mathrm{P} 1=0.325$. Se lleva a cabo el contraste de hipótesis donde $\mathrm{nc}=12$ y $\mathrm{n}+=28$ por lo tanto la media es 17.8 y la desviación típica 0.493 . Realizando el contraste de hipótesis aparecen evidencias para rechazar la hipótesis nula. Se puede concluir que la secuencia no es aleatoria.

Se efectúa este análisis de las cuatro secuencias para explicar cómo existe una relación directa entre las simulaciones y el test de aleatoriedad. Así, basta con que una de las características de una secuencia presente valores por encima de P99 o por debajo P1 para que no se verifique la aleatoriedad de la secuencia. Esto es lo que parecer ser que debe ver un alumno de forma intuitiva. ¿Quizás no es demasiado difícil saber dónde están los valores límites? ¿No se necesitaría haber realizado un estudio previo similar al llevado a cabo en este documento?

\section{DISCUSIÓN Y CONCLUSIONES}

Se deben comentar los resultados obtenidos en el estudio respecto a otros estudios previos citados en el apartado de introducción.

Es importante que los maestros sepan identificar cuál es el tipo de razonamiento que deben llevar al aula, para adecuarse a sus objetivos de sus enseñanzas. Para validar la mejor estrategia es conveniente analizar empíricamente un resultado y apoyar una decisión o plantear juegos con argumentos lógicos y probados.

Como conclusiones finales en el lanzamiento de una moneda hasta obtener una secuencia con los sucesos elementales iguales o cercanos a la probabilidad teórica, se ha encontrado lo siguiente: 
-No tiene por qué haber un número equilibrado entre el número de caras y cruces, sobre todo si no hay un número grande de lanzamientos.

-Las longitudes de rachas en el $90 \%$ de las secuencias son de 4 o menos repeticiones seguidas de caras o de cruces, en el $95 \%$ son de 5 o menos, sólo hay un $1 \%$ de secuencias que son mayores de 7 (P99=7).

-El número de rachas se encuentra muy cercano a la mitad de los lanzamientos realizados independientemente del tamaño de la secuencia.

Es importante que los maestros conozcan estas conclusiones. Después de este estudio se puede concluir que el resultado del lanzamiento de monedas no es para nada intuitivo. Es preciso hacer un estudio previo y seguidamente poder argumentar en base a éste.

\section{REFERENCIAS}

Arteaga, P., Ortiz, J.J., y Batanero, C. (2008). Uso de gráficos comparación de dos distribuciones por futuros profesores. Poster presentado en la 22 Reunión Latinoamericana de Matemática Educativa (RELME), 22. México.

Bar-Hillel, M., y Wageraar, W.A. (1991). The perception of randomness. Advances in Applied Mathematics 12, 428-454.

Batanero, C., y Serrano, L. (1999). The meaning of randomness for secondary school students. Journal for Research in Mathematics Education, 30(5), 558-567.

Carrera, E. (2002). Teaching statistics in secondary school. An overview: From the curriculum to reality. En B. Phillips (Ed.), Proceedings of the Sixth International Conference on Teaching of Statistics. Ciudad del Cabo: IASE. CD ROM.

Fischbein, E. (1987). Mathematics education library. Intuition in science and mathematics: An educational approach. Netherlands: Reidel Publishing Company.

Green, D.R. (1983). A survey of probability concepts in 3000 pupils aged 11-16 years. En D.R.

Grey., P. Holmes., V. Barnett., y G.M. Cosntable. (Eds), Proceedings of the ICOTS 1. Vol. 2 (pp.766-783). Sheffiend, UK: Teaching Atatistics Trust.

Green, D.R. (1990). Using computer simulation to develop statistical concepts. Teaching Mathematics and Its Applications, 9(2), 58-62.

Green, D.R. (1991). A longitudinal study of pupil's probability concepts. Loughborough: University of Loughborough.

Kahneman, D., Slovic, P., y Tversky, A. (1982). Judgement under uncertainty: Heuristics and Biases. Cambrigde: Cambridge University Press.

Konold, C. (1995). Confessions of a coin flipper and would-be instructor. The American Statistician, 49(2), 203-209.

Recibido: 5 de agosto de 2019

Recepción Modificaciones: 19 de agosto de 2019

Aceptado: 6 de septiembre de 2019 